\title{
Studying the Concentration Field Distribution in Shear Concentration Convective Flows of a Viscous Incompressible Fluid in a Plane Horizontal Layer with Immobile Boundaries
}

\author{
N. V. Burmasheva ${ }^{1,2, \text { a) }}$ and E. Yu. Prosviryakov ${ }^{1,2, b)}$ \\ ${ }^{1}$ Institute of Engineering Science, Ural Branch of the Russian Academy of Sciences, \\ 34 Komsomolskaya St., Ekaterinburg, 620049, Russian Federation \\ ${ }^{2}$ Ural Federal University named after the first President of Russia B.N. Yeltsin, \\ 19 Mira St., Ekaterinburg, 620002, Russian Federation \\ ${ }^{a)}$ Corresponding author: nat_burm@mail.ru \\ b)evgen_pros@mail.ru
}

\begin{abstract}
Depending on the reasons causing changes in the density of a fluid, it is customary to distinguish between thermal convection and concentration-induced convection. The phenomenon of concentration-induced convection often accompanies the flow of a working fluid in devices working, for example, with fluid coolants. When modeling such flows, the consideration of the generalization of the Boussinesq hypothesis assuming the dependence of the fluid density on the impurity concentration and on the temperature of the fluid itself leads to the situation that the flow velocity field and the concentration field affect each other. The paper investigates the features of the shear convective flow of a viscous incompressible fluid with an admixture in the horizontal layer. The system of concentration-induced convection equations consisting of the equation of motion of a viscous fluid, the concentration change equation, and the incompressibility condition is taken as a system of defining relations. The solution is sought in the class of functions linear in two coordinates $(x$ and $y)$. As the boundary conditions, it is assumed that the no-slip condition is met at the lower impermeable boundary, that the upper boundary of the layer is immobile, and that the distribution of salinity and pressure is specified on it. A complete solution of the boundary value problem is given. The attention is focused on the analysis of the distribution of the concentration field inside the fluid layer. It is shown that, according to the solution of the boundary value problem for the concentration field, its homogeneous (with respect to the horizontal coordinates) component does not vanish inside the layer. The components of the concentration field, linearly dependent on the horizontal coordinates, take a constant value everywhere inside the layer. The change in the mutual arrangement of the isolines of the concentration field at the transition from one section of the fluid layer to another is studied. Relevant findings are illustrated.
\end{abstract}

\section{BOUNDARY VALUE PROBLEM FORMULATION}

The steady-state shear convective flow of a viscous incompressible fluid, induced by the presence of impurity in the fluid is described by a system of equations including the Navier-Stokes equations in the Boussinesq approximation, the incompressibility equation, and the equation for the change in the concentration of the light phase of a binary mixture [1],

$$
\begin{gathered}
(\boldsymbol{V} \cdot \nabla) \boldsymbol{V}=-\nabla P+\nu \Delta \boldsymbol{V}+g \beta C \boldsymbol{k} ; \\
(\boldsymbol{V} \cdot \nabla) C=d \Delta C ; \\
\nabla \cdot \boldsymbol{V}=0 .
\end{gathered}
$$


Here, $\boldsymbol{V}=\left(V_{x}(x, y, z), V_{y}(x, y, z), 0\right)$ is the velocity vector; $P(x, y, z)$ is the deviation of pressure from hydrostatic, divided by the average density; $C(x, y, z)$ is the concentration of the light phase of a binary mixture; $v, d$ are the kinematic (molecular) viscosity of the fluid and the diffusion coefficient, respectively; $g$ is the acceleration of gravity; $\nabla, \Delta$ are the Hamilton and Laplace three-dimensional operators, respectively.

The solution is sought in the class [2-5]

$$
\begin{gathered}
V_{x}=U(z) ; V_{y}=V(z) ; V_{z}=0 ; \\
P=P_{0}(z)+x P_{1}(z)+y P_{2}(z) ; C=C_{0}(z)+x C_{1}(z)+y C_{2}(z) .
\end{gathered}
$$

Note that the nonlinear system of partial differential equations (1) includes five scalar equations for four unknown functions, namely velocities $V_{x}, V_{y}$, concentration $C$, and pressure $P$. This means that system (1) is overdetermined. However, for the velocity class of the form represented by Eq. (2), the incompressibility equation is satisfied identically; thus, the balance between the number of equations and the number of unknowns for class (2) is satisfied.

It can be rigorously shown that, by substituting the selected representation of the hydrodynamic fields (2)-(3) into system (1), we can reduce this system to the following equivalent system of ordinary differential equations for the components of the expansion (2)-(3):

$$
\begin{gathered}
C_{1}{ }^{\prime}=0 ; C_{2}{ }^{\prime}=0 ; P_{1}{ }^{\prime}=g \beta C_{1} ; P_{2}{ }^{\prime}=g \beta C_{2} ; \\
v V^{\prime \prime}=P_{2}, v U^{\prime \prime}=P_{1}, d C_{0}{ }^{\prime \prime}=U C_{1}+V C_{2}, P_{0}{ }^{\prime}=g \beta C_{0} .
\end{gathered}
$$

The prime in system (4) denotes differentiation with respect to the vertical (transverse) coordinate $z$. The equations of system (4) are integrated according to the given order.

In order to describe real flows by solutions of system (4), the conditions describing the behavior/state of hydrodynamic fields at the boundaries of the flow region must be additionally specified. Moreover, the number of conditions must coincide with the order of the system of defining relations. Since system (4) is a thirteenth-order system, it is necessary to formulate thirteen boundary conditions.

As an example, we further consider the steady-state diffusion flow of a viscous binary fluid in an infinite horizontal layer of a given thickness $h$. Assume that the solid boundary $z=0$ of the fluid layer is impermeable and that the fluid no-slip condition is fulfilled on it [6-8],

$$
\left.V_{x}\right|_{z=0}=\left.V_{y}\right|_{z=0}=0,\left.\frac{\partial C}{\partial \boldsymbol{n}}\right|_{z=0}=0 .
$$

Here, $\boldsymbol{n}$ is the normal to the lower boundary of the layer.

Assume that the pressure distribution at the upper fixed boundary $z=h$ is homogeneous and that the distribution of impurity concentration linearly depends on the longitudinal (horizontal) coordinates,

$$
\left.V_{x}\right|_{z=h}=\left.V_{y}\right|_{z=h}=0,\left.P\right|_{z=h}=S_{0},\left.C\right|_{z=h}=a x+b y .
$$

\section{EQUATION SYSTEM SOLUTION}

The solution of the boundary value problem (4)-(6) is polynomial and written as follows:

$$
\begin{gathered}
U=\psi Z(-2+Z)(-1+Z) ; \\
V=\gamma Z(-2+Z)(-1+Z) ; \\
C_{1}=a ; C_{2}=b ; \\
C_{0}=\lambda(-1+Z)\left(8+8 Z+8 Z^{2}-12 Z^{3}+3 Z^{4}\right) ;
\end{gathered}
$$




$$
\begin{gathered}
P_{1}=\xi_{1}(-1+Z) ; P_{2}=\xi_{2}(-1+Z) ; \\
P_{0}=S_{0}+\xi_{3}(-1+Z)^{2}\left(11+6 Z+Z^{2}-4 Z^{3}+Z^{4}\right) .
\end{gathered}
$$

Here,

$$
\begin{gathered}
Z=z / h, \psi=a g \beta h^{3} /(6 v), \gamma=b g \beta h^{3} /(6 v), \lambda=\left(a^{2}+b^{2}\right) g h^{5} \beta /(360 d v), \\
\xi_{1}=a g \beta h, \xi_{2}=b g \beta h, \xi_{3}=\left(a^{2}+b^{2}\right) g^{2} h^{6} \beta^{2} /(720 d v) .
\end{gathered}
$$

In what follows, the impurity concentration field is analyzed in detail.

\section{INVESTIGATION OF THE CONCENTRATION FIELD}

It can be seen from solution (7) that the longitudinal gradients $C_{1}, C_{2}$ are constant functions; therefore, all the features in the distribution of the concentration field depend on the properties of the background concentration $C_{0}$, measured relative to the concentration level at the upper boundary of the layer. Let us study these properties.

Up to the constant factor $\lambda$ in solution (7), the behavior of the background concentration coincides with the behavior of a fifth-degree polynomial

$$
f(Z)=\lambda(-1+Z)\left(8+8 Z+8 Z^{2}-12 Z^{3}+3 Z^{4}\right) .
$$

Herewith, the factor $\lambda$ can take only non-negative values at any values of the parameters $a$ and $b$ determining the distribution of the concentration field at the upper boundary of the layer.

The polynomial $f$ vanishes at $Z=1$. This is consistent with the fact that the value of concentration at the upper boundary is taken as the reference level. If this polynomial vanishes at other points inside the layer, the background concentration at these points takes values coinciding with the values of the impurity concentration at the upper boundary of the layer. The graph of the function $f$ is shown in Fig. 1.

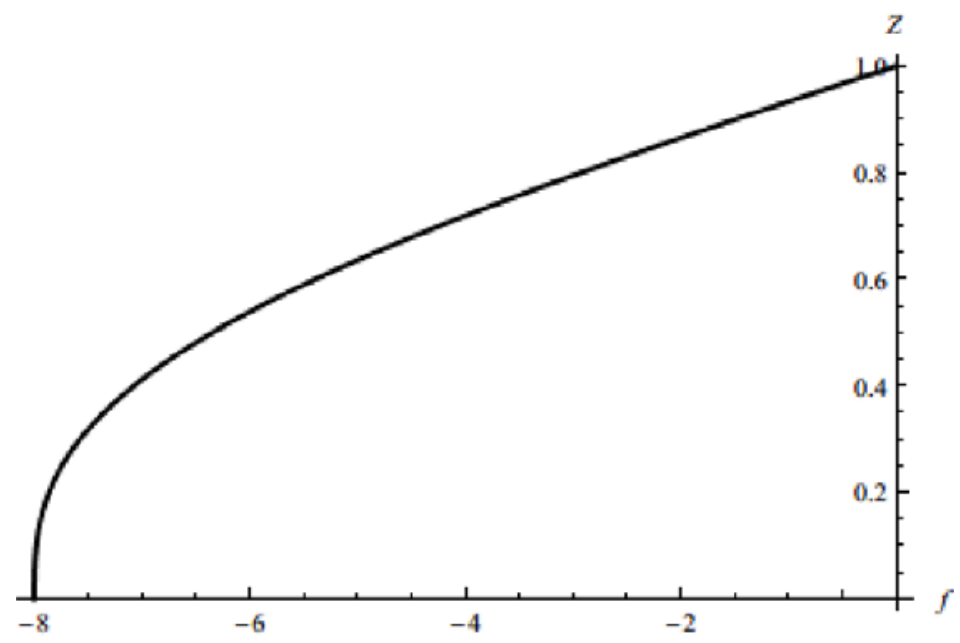

FIGURE 1. The behavior of the function $f(Z)$

Figure 1 illustrates the fact that the function $f(Z)$ is a monotonically increasing one, and it takes only negative values on the interval $(0,1)$. This means that the impurity concentration in the layer is unevenly distributed its highest value being observed at the upper boundary of the fluid layer. In addition, the exact solution (7) for the background concentration of $C_{0}$ does not allow the appearance of stratification points [9-14]. 
Note that, since the components $C_{1}, C_{2}$ are constant by virtue of (7), the addition of the fields $C_{1}(Z) x$ and $C_{2}(Z) y$ to the background concentration $C_{0}$ does not change the shape of the profile of the total field $C$ as compared to the shape of the profile of the field $C_{0}$, but only determines the shift of this profile relative to the vertical axis OZ, Fig. 2.

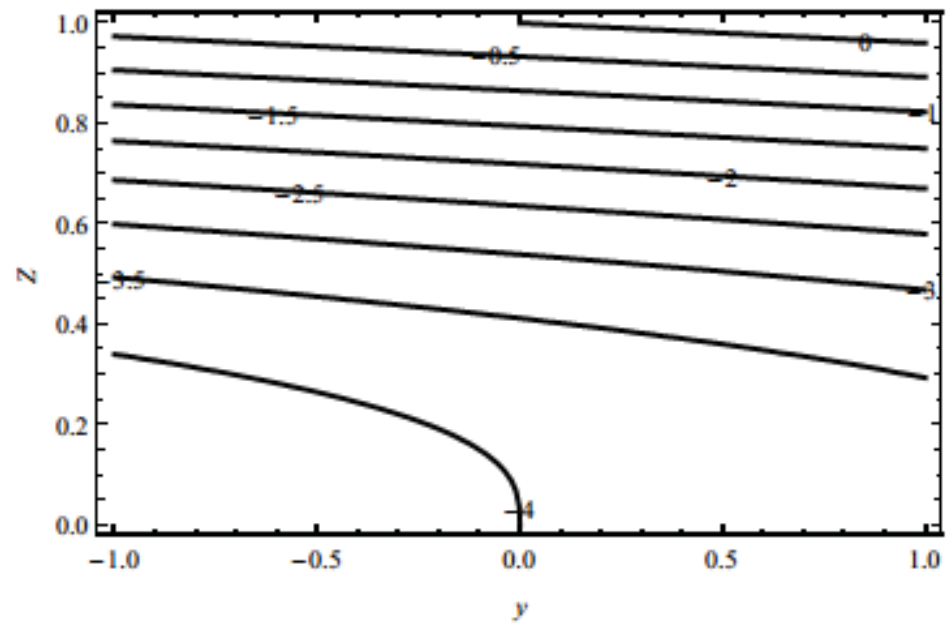

FIGURE 2. Level lines of the concentration field in the cross section $x=0$ for $\lambda=0.5, a=0.1$, and $b=0.3$

\section{CONCLUSION}

The exact solution describing the convection of a viscous incompressible fluid in a horizontal layer, induced by inhomogeneous distribution of impurity at one of the boundaries of the layer has been obtained. The distribution of the concentration field has been studied in detail.

\section{REFERENCES}

1. G. Z. Gershuni and E. M. Zhukhovitskii, Convective Stability of Incompressible Fluids (Wiley/Keter Press, Jerusalem, 1976).

2. M. Couette, Ann. Chim. Phys. (Ser. 6) 21, 433-510 (1890).

3. N. V. Burmasheva and E. Yu. Prosviryakov, AIP Conf. Proc. 2176, 030002-1-030002-5 (2019).

4. N. V. Burmasheva, E. A. Larina, and E. Yu. Prosviryakov, AIP Conference Proceedings 2176, 030023 (2019).

5. N. V. Burmasheva and E. Yu. Prosviryakov, Vestn. Samar. Gos. Tekhn. Univ., Ser. Fiz.-Mat. Nauki 21(1), 180-196 (2017).

6. S. V. Goldstein, Modern Developments in Fluid Mechanics (Oxford Univ. Press, Oxford, 1938).

7. L. Prandtl and O. Tietjens, Fundamentals of Hydro- and Aeromechanics (McGraw-Hill Book Company, New York - London, 1934).

8. E. S. Baranovskii and M. A. Artemov, Vestnik Sankt-Peterburgskogo Universiteta. Prikladnaya Matematika. Informatika. Processy Upravleniya 13(4), (2017).

9. G. A. Ostroumov, Free convection under the condition of the internal problem (National Advisory Committee for Aeronautics, Washington, NACA Technical Memorandum 1407, 1958).

10. R. V. Birikh, J. Appl. Mech. Tech. Phys. 7(3), 43-44 (1966).

11. P. Riesco-Chueca, J. F. De la Mora, Journal of Fluid Mechanics 214, 639-663 (1990).

12. V. Shtern, Counterflows: Paradoxical Fluid Mechanics Phenomena (Cambridge University Press, Cambridge 2012).

13. J. M. Dorrepaal, Journal of Fluid Mechanics 163(1), 141-147 (1986).

14. J. T. Stuart J. T., Journal of the Aerospace Sciences 26(2), 124-125 (1959). 